\title{
Human-Computer Interfacing for the Severely Physically Disabled
}

\author{
A.D. Cherry*, M.S. Hawley\#, M. Freeman** and P.A. Cudd* \\ *Dept. Electronic and Electrical Engineering, University of Sheffield, Sheffield, S1 3JD \\ **Philippa Cottam Communication Clinic, Dept. of Speech Science, University of Sheffield, \\ Sheffield, S10 2TA \\ \#Dept. Medical Physics, Barnsley District General Hospital NHS Trust, Barnsley, S75 2EP
}

\begin{abstract}
Human computer interfacing issues and the need for design standards for switch operated integrated rehabilitation systems are discussed. Input methods and compatibility problems are summarised. Formulae for access times to menu structures for a number of single switch selection methods are given. Quantitative comparisons are given for example selection processes. It is concluded that row column scanning is fastest for small selection sets. Advantages and disadvantages of the use of multiple switches for input are indicated.
\end{abstract}

\section{Introduction}

Rehabilitation integrated systems are in their infancy, in the future as they mature they offer the potential to meet the electronic device control needs of a broad range of disabled people. The latter electronic devices can support some or all of the following functions: mobility, communication and environment control, and especially when computer based, education and employment. The potential for computer based integrated systems to benefit disabled children is significant because there is easier and greater scope for the system to grow with the child than with Rehabilitation Systems of the past.

The important features which are offered by a properly designed integrated system are: appropriateness to a large target population; an user interface that can be optimised to the individual's needs and abilities, including the adaptability to cater for the changing needs of the user; safe operation; a comprehensible and functional setting up procedure. Other factors are cost, robustness and compactness. On most of these points, integrated systems based on portable computers appear to show considerable promise.

Designed integrated/semi-integrated systems are emerging ${ }^{1,2}$, but agreed design standards are not yet in existence. Words $+^{3}$ produce a suite of applications that can be used together as an integrated system. However, the user is restricted to using software from that company and would have difficulty using it with other software successfully. A major advantage of using Words+ software is the availability of free upgrades for life. Prentke Romich ${ }^{4}$ build stand alone communication aids which can be enhanced to provide environmental control and computer keyboard emulation. $\mathbf{A}$ number of systems are evolving 5,6 but these are not yet sufficiently established to be widely used for the development or provision of integrated systems. The advent of 
Microsoft Windows has established a better software design environment for the PC. It should be noted that other platforms with similar graphical user interfaces offer this advantage, e.g. Apple Macintosh platforms.

The Sheffield and Barnsley Rehabilitation Research Group have developed integrated systems designed for severely physically handicapped people using a number of switches for the input device ${ }^{7,8}$. More recently systems based on fully PC compatible computers, e.g. BASIS $^{9}$, have offered colour displays and the option for use of pointing devices for input.

This paper discusses some of the factors that must be considered in the development of switch user interfaces for integrated systems. Many switch users operate their computer by use of mouse and/or keyboard emulators. The use of an icon and switch based user interface, i.e. BASIS, has caused the authors to reconsider the suitability of standard input device emulators for generic applications.

\section{Input Methods}

For users with insufficient motor control to use a standard computer keyboard and/or mouse, a number of alternative devices exist. They generally fall into one of three categories:

- Alternative keyboards. These range from keyboards with alternative layouts to expanded keyboards with either large or very tightly-packed buttons, or, switch operated emulators. Usually the application software will use its standard keyboard interfacing.

- Mouse emulators. Touch screens, track balls, head pointers etc. come into this category. Usually the application software will use its standard mouse interfacing.

- Switches. Switches of varying size and required operating force are used.

They require dedicated switch driven interfacing.

Depending on which of these alternatives is being used, the method of connecting them varies. Alternative keyboards usually connect to the keyboard port. Mouse emulating devices usually connect to the serial port, often requiring a driver in addition to the mouse driver. The situation for switches, however, is more complex. Unlike the keyboard or mouse, there is currently no standard means of connecting switches to a computer. Typical switch connection techniques are as follows:

- Switches are hardwired in parallel to the buttons on a mouse.

- A switch in circuit between a common line and a handshaking line on the serial port.

- A switch in circuit between a common line and a handshaking line on the parallel port.

- A dongle is connected to a standard port, the switches are connected to the dongle. This provides transparency (e.g. a 'through' printer port) and software copy protection. 


\subsection{Software compatibility of integrated systems using switch input}

If a switch user wishes to use just one switch-driven application, no problems should occur. Increasingly however, computers are being used as the basis of integrated systems with a number of both assistive and 'standard' applications needing to be controlled from the switches. Each application may have a different switch interface, and indeed require a different port connection. Applications not designed for switch use usually require a keyboard and/or mouse emulator to function. With such an emulator, switch presses are transformed by the emulator to key presses or mouse operations to create input that the application can accept. Problems associated with using multiple applications can be eased in environments such as Microsoft Windows, but compatibility between the applications' switch inputs is still not guaranteed. A typical integrated system may consist of products from two or three different sources and the above problems can still exist. The user is then left with the following choices:

- A carer may have to move the switch connection for the user;

- The user may have to use a compatible but possibly inferior application;

- Technical assistance may have to be sought, the problem may or may not be solvable by local expertise. If not, one or more of the applications would have to be re-designed.

Clearly none of the above are satisfactory solutions. The first two points result in the user having an inferior integrated system. The third point may require great effort and take too long. The problems are exacerbated if these multiple applications control a speech synthesiser, infra-red sender and wheelchair. With desktop computers the required extra ports can be added via an expansion card, but notebook computers, used commonly by wheelchair-bound users have little or no means of expansion.

\subsection{Standardisation}

The well specified user interface in modern environments, such as Windows with its 'feel of similarity' across applications, is a step forward in potentiating access to applications for disabled individuals. However, it does not go far enough. The philosophy behind many of the commercially available graphical user interfaces includes the tenet 'the user should need to know a minimum, preferably nothing, about the computer hardware to use the computer', leading to the trend that more and more of the low-level system operation, such as the code that interprets the incoming signals from the mouse, is difficult to replace. Access to the low-level system operation is of fundamental importance to Rehabilitation system developers. The authors would argue that commercially available user interfaces should hide all unnecessary hardware related aspects to facilitate ease of use, but that the blocks of code handling the user input and feedback be replaceable, i.e. make these blocks separate applications. For switch input, generic software standards that interpret the input and possibly a standard hardware port are needed. The exact nature of the hardware standard is not so certain because this could also be specified for the PCMCIA interface and/or the serial, parallel, PS2, keyboard or perhaps games ports. 
The future requirements for the hardware standard are not sufficiently understood due to safety issues and the possibility of needing adaptive interfaces.

The M3S bus project ${ }^{6}$ proposes a standard hardware interface to which all assistive devices belonging to an integrated system would be attached. As this interface would have to be incorporated into all new equipment including computers its general acceptance could take a long time. Also, following its rationale fully implies even providing each switch with an M3S interface. Ultimately, international standards and approaches are desirable for this technology to mature. The open system approach offered by Comspec 5 is to be welcomed. Just like other rehabilitation development, standards should be derived from the needs of the users. The needs of the users will determine the required human computer interface which in turn will specify the requirements for the software and hardware. In considering the definition of the standards, the goal should be maximising functionality for the user. It often looks as if software is written with too much emphasis on the provider; of course, if systems do not have a simple set up procedure, they are unlikely to be supplied. Integrated system and general assistive devices developers should aim to at least provide clearly specified hardware/software interfacing information.

\section{The Human Computer Interface}

Two of the most important factors of the human-computer interface are the method of presentation of information to the user and the efficiency with which the user is able to achieve' tasks.

\subsection{Presentation of information}

It is generally agreed that icon-based interfaces are more effective than purely textual ones. This has lead to the wealth of graphical user interfaces currently used. In a rehabilitation context, the use of symbols in complex selection tasks in communication aids is common. It is possible to view the use of symbols in an integrated system as an extension of this. As a result, the BASIS system developed by the authors employs icons for presentation of information to the user. Colour screens are able to convey more information than monochrome and so are suitable for more complex set-ups, and, for systems for children and those with perceptual or cognitive difficulties. Figure 1 defines the various parts of a menu. An item is in the case of BASIS a picture (icon) depicting the control to be initiated.

The style of highlighting in menus where items are selected via scanning is important. Items or groups of items highlighted should not be obscured or their picture quality degraded by the highlight itself. If this is the case, the user may have to remember the function of the items obscured, possibly requiring too great a cognitive demand. Showing an item has been selected is important, especially in some control applications where it may not be obvious the action has been initiated.

The presentation and organisation of the selection sets will affect operating efficiency. When designing an interface, the cognitive and perceptual demands of the interface must be taken into account. The choice in communication applications between dynamic menus and those using multi-meaning symbols is currently the 
subject of debate. Each method is supportable in BASIS and is of use in particular

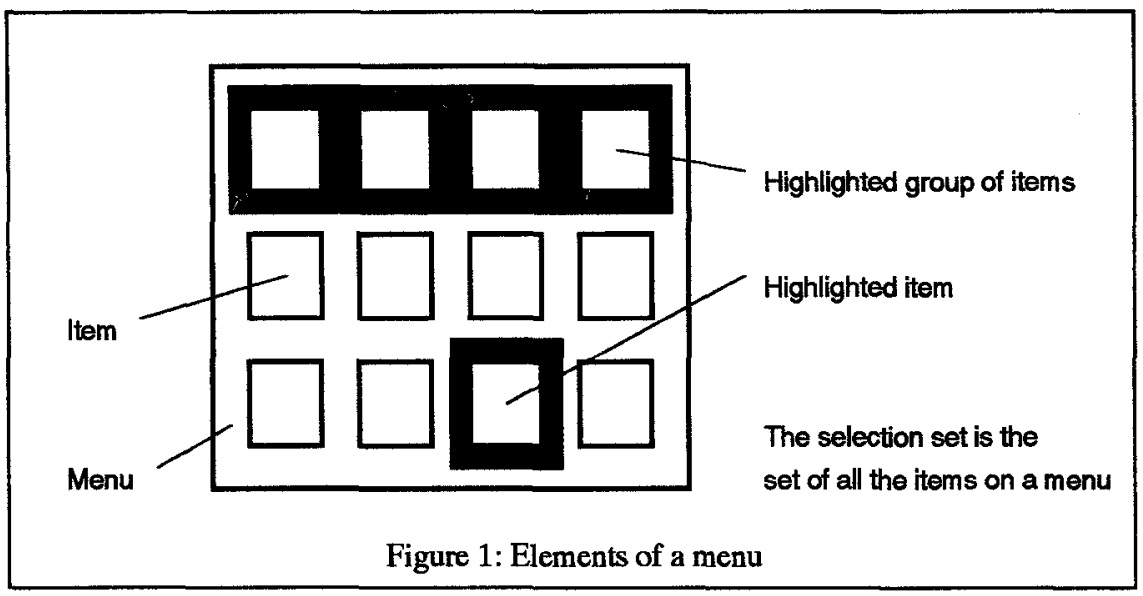

situations. However, the BASIS system was designed to work with the principle of dynamic displays. This method produces the lesser cognitive load on the user since selection depends on recognition and does not greatly load the user's memory. Since BASIS is primarily aimed at children, a lower cognitive loading is appropriate.

\subsection{Selection Processes with switches}

Making switches send key presses via a keyboard emulator is not always efficient. A keyboard emulator offers the user the choice of any of the keys available on the keyboard during the selection process, whereas an effective generic switch user interface would only offer the choice of selecting the relevant commands at any given point in time. This would include all of the keys of a keyboard when that was appropriate.

BASIS supports a number of different input selection devices, including mouse emulators and switches. Most switch controlled applications give a choice of a small number of scanning methods. Currently the provider and user have no means of deciding which is the best selection method; they are often chosen according to the provider's preferred method. The results below compare the efficiency of some different scanning selection processes. The scanning methods compared were :

\section{A. Row-column.}

B. Binary division - split selection set in half and scan, on selection split remaining items and scan, this continues until the required item is left

C. Single division \& row column - binary divide the selection set once, proceed with row-column scanning the remaining half.

D. Double division \& row column - binary divide the selection set twice, proceed with row-column scanning.

Figure 2 shows an example of scanning method $\mathrm{C}$. 
a)

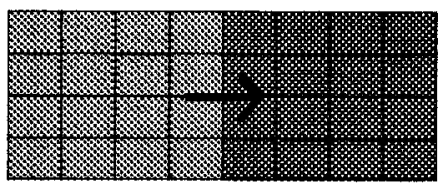

b)
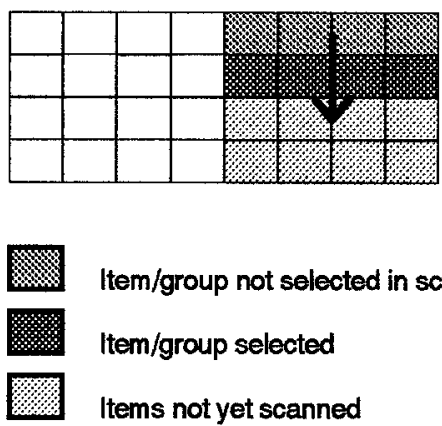

Item/group not selected in scan Item/group selected

Items not yet scanned c)

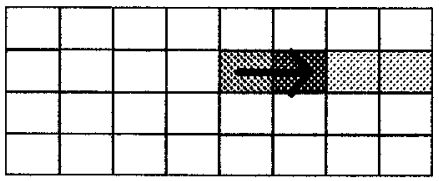

d)

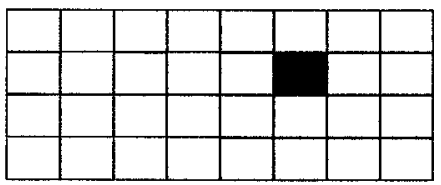
Items no longer offered

Selected item

Figure 2: Example of single division \& row-column scanning

Average times for selection were calculated for each of the methods with a number of menu sizes ranging from a $2 \times 2$ to a $16 \times 8$ matrix . The average times were calculated assuming one-switch input with a $1 \mathrm{~Hz}$ timer moving the scan highlight. In this theoretical test, the following assumptions were made:

- scan always starts from the same position (i.e. top left option offered);

- all items have equal probability of being selected;

- user knows menu layout and required item is correctly selected first time;

- selection is made as soon as that item is highlighted.

The table below shows the formulae derived for the average time to access an item using each scanning method. It also shows the number of switch presses required to select an item. $\mathrm{n}_{\mathrm{x}}$ and $\mathrm{n}_{\mathrm{y}}$ are the number of items on the menu page horizontally and vertically respectively.

\begin{tabular}{|c|c|c|}
\hline Type & Average access time & Num. switch presses \\
\hline $\mathrm{A}$ & $\frac{1}{2}\left(2+n_{x}+n_{y}\right)$ & \\
\hline $\mathrm{B}$ & $\frac{3}{2} \log _{2}\left(n_{x}+n_{y}\right)$ & $\sqrt[3]{n_{x} n_{y}}$ \\
\hline $\mathrm{C}$ & $\frac{16+n_{x}+n_{y}}{4}$ & 3 \\
\hline $\mathrm{D}$ & $\frac{10+n_{x}+2 n_{y}}{4}$ & \\
\hline
\end{tabular}

For menus with $n_{x} \leq 6$ and $n_{y} \leq 6$ items, row column scanning is with the above assumptions the fastest access method. For menus larger than this, division methods 
$C$ and $D$ have significant time advantages. For menus with $n_{x} \geq 8$ and $n_{y} \geq 8$, method $\mathrm{D}$ is fastest. Binary division (method B) is always slower than the alternatives and has the added disadvantage of requiring many switch presses to select a particular item.

This study indicates that row column scanning is fastest for single switch operation of a small menu of items. Extension of this study to other scan methods, dynamic displays and larger numbers of switches is in progress.

There are few rehabilitation aids which currently allow input from more than two switches. It is apparent that employing a greater number of switches could increase selection efficiency. Using switches in non-scanning selection methods and the fact that multiple switches may be utilised to associate the desired action with the switch positions could be advantageous. A disadvantage is that the physical and cognitive limitations of a user may preclude the use of more than one switch. However, the possibility of more efficient access for some makes it worthy of further study.

Short term and long term adaption of the display may also help to improve efficiency. Short term adaption is temporary reorganisation of the items presented in a menu dependent on current usage. At a simple level this could entail presenting to the user only those items that can follow the currently selected item. Short term adaption could also take into account the user tiring over the course of a day allowing them to change from their favoured switch selection mechanism to a less demanding and slower one. Long term adaption involves establishing a pattern of selections and introducing more efficient selection routes where appropriate.

\section{Conclusions}

A very important part of any proposed electronic aid is the Human-device interface, generic rehabilitation integrated systems require sophisticated background interfacing. User interfacing is needed that allows flexible configuration and appropriate support of communication, control and software tasks. The required flexibility in future integrated systems and their user interfaces implies that there is still much research and development to do. The latter development must include 'system' configuration tools that enable rehabilitation aid providers to supply and support systems.

Currently not enough is known about the human computer interfacing of integrated systems for disabled people to provide an optimally functional aid. The authors have identified that only limited quantitative data exists on efficiency of switch accessing methods and to a lesser extent on visual feedback. Most study of human computer interfacing in this context has been for communication aids 10,11 . The work presented throws some light on efficiency of switch use for a static display. This work is being extended to more complex systems. As has been highlighted by the above discussion, standards need to be established both for hardware and software aspects of computer based integrated systems. Any standards proposed should not stifle innovation. Modern graphical user interfaces for the able-bodied market offer significant improvements in terms of access to standard applications through such packages as Switch Access to Windows ${ }^{12}$. Although many of the 
software applications specifically developed for the rehabilitation market have yet to be implemented on Microsoft Windows, this process should speed up since compilers will be available shortly which do not require knowledge of the workings of the Windows environment.

Current computer user interfaces and environments are still limiting because most are tied to a particular software application, hardware or fixed user interface operating environment. The challenging next step is to allow an individuals interfacing requirements to be specified to re-configure the standard user interface for computer systems. The authors would argue that individual user interface configuration is achievable if the concept behind the human-computer interfacing of state of the art user interfaces is extended. It is the intention of the authors to investigate this.

\section{References}

1. Bühler C, Uniform user interface for communication and control, Proc. Ecart 2 Stockholm, pp. 22.3, May 26-28 1993

2. Redmond B., Allen R., Bullock R, Mobile Communications and Environment Control for Wheelchair Users (MECCS), Rehabilitation Technology, ed. E. Ballabio, IOS Press, Amsterdam, pp. 65-69, 1993

3. Words+ Inc., PO Box 1229, Lancaster, CA 93534, USA.

4. Prentke Romich Company, 1022 Heyl Road, Wooster, Ohio 44691, USA.

5. Lundälv M., Svanæs D, Comspec - towards a modular software architecture and protocol for AAC devices, Rehabilitation Technology, ed. E. Ballabio, IOS Press, Amsterdam, pp. $55-59,1993$

6. van Woerden J.A. et al., A safe and easy to use integrated control and communication method M3S, Rehabilitation Technology, ed. E. Ballabio, IOS Press, Amsterdam, pp. 75 79,1993

7. Hawley M.S., Cudd P.A., Wells J.H., Wilson A.J., Judd, P., Wheelchair-mounted integrated control systems for multiply handicapped people, J. Biomedical Engineering, vol. 14, iss. 5, pp. 193-198, May 1992

8. Cudd P.A., Hawley M.S., Inexpensive PC-based integrated control systems for severely physically disabled people, Proc. 14th Ann Int. conference of the IEEE Engineering in Medicine and Biology Soc., Paris, pp. 1648-9, 1992

9. Hawley M.S., Cudd P.A., Cherry A.D., Implementation of a PC-based integrated control system for children, to be published in J. Biomedical Engineering, vol. 16, iss. 5, May 1994

10. Rosen M.J., Goodenough-Trepagnier C., Modelling Reciprocal Tapping Performance Of Neurologically Disabled People For Prediction Of Communication Rate, Proc. ASME, pp, Nov. 1987

11. French, L.E., Reddy, N.P., Communication and errors in single switch controlled autoscanning communication aids, Annual International Conference of the IEEE Engineering in Medicine and Biology Society, vol. 13, iss. 4, pp. 1837-8, 1991

12. Head P., Poon P., Morton C., Colven D., Lysley A, Switch Access To Windows 3 (SAW) A New Concept In Emulation Techniques, Proc. Ecart 2 Stockholm, pp. 22.2, May 26-28 1993 